\title{
Solar Shadow Positioning Algorithm
}

\author{
Wanqiao Wang \\ School of North China Electric Power University, Baoding 071000, China; \\ qiao.11@qq.com
}

Keywords: solar shadow, Positioning, Algorithm, MATLAB.

\begin{abstract}
How to confirm the photographing location and the photographing date is an important aspect of video data analysis. Solar shadow positioning technique is a method to confirm the photographing location and date through analysis of solar shadow changes of objects in videos. According to the image-forming principle, the ratio of the measured coordinate value to the straight-bar height is equal to the ratio of the practical coordinate value to the straight-bar height. Therefore, videos can be imported into MATLAB to capture images at the required time interval. CAD can be used to measure the slanted coordinate of shadow points in images. Through coordinate transformation, the right-angle coordinate value in images can be confirmed. According to the proportional relation, the coordinate of the vertex of the straight-bar shadow can be confirmed according to the proportional relationship. Based on that, the potential photographing location can be confirmed according to models. To analyze the photographing location and date of videos, the coordinate of the vortex of the practical straight-bar shadow can be put into the model Question 3 to confirm the potential photographing location and date.
\end{abstract}

\section{Introduction}

According to Geometrical Optics, light travels in straight lines in the even medium. Shadows are caused by the shading of light by certain object. Non-transparent objects impede light transmission, thus forming dark regions, which can be called shadows. (Light is a collection of visible rays.) Shadow changes are mainly decided by the straight-bar height, time and solar position. Under the condition that the straight-bar height is own, changing rules of solar shadows of the straight bar can be confirmed by analyzing changes of the latter two. Time can be described by time difference and true solar time. Solar position can be described by solar declination, solar altitude and solar azimuth [1]. Their relationship is shown in Fig. 1:
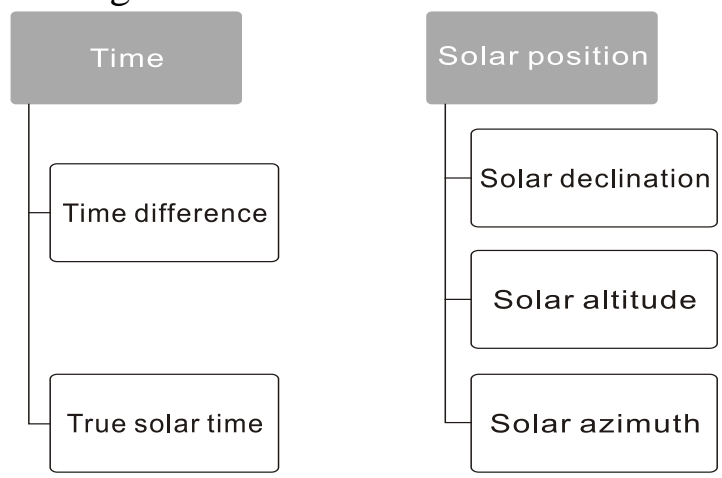

Fig.1 Relation diagram

\section{Time}

2.1 Time difference, EQ

EQ refers to the difference between the true solar time, TT, and the local solar time (local time), TM, which can be calculated according to the following equation:

$$
E_{Q}=0.0028-1.9857 \sin Q+9.9059 \sin 2 Q-7.0924 \cos Q-0.6882 \cos 2 Q
$$




$$
Q=2 \pi \times 57.3\left(N \times \Delta N-N_{0}\right) / 365.2422
$$

Where,

1) $\mathrm{N}$ stands for the day ranked according to the number of days. January 1 is 0 ; January 2 is 1 ; and the rest can be deduced by analogy. $\mathrm{N}$ of December 31 is 364 for the common year and 365 for the leap year.

2) $\Delta N$ stands for the rectified $\mathrm{N}[2]$ :

$\Delta N=(W-L) / 24$

Where,

a) L stands for the rectified value of the time difference between the observation location and the Greenwich longitude. (Negative for the east longitude; positive for the west longitude. China is located within the east longitude, so $\mathrm{L}$ is negative.)

$\pm L=(D+M / 60) / 15$

(D: Measurement value of the observation location's longitude; M: Numerator of the measure observation location's longitude)

b) $\mathrm{W}$ stands for the rectified value of the time difference between the observation moment and Greenwich Zero-Hour.

$W=S+F / 60$

(S: Observation time; F: Numerator of the observation moment)

2.2 Solar position

In order to directly show the measurement methods of solar altitude and solar azimuth, CAD schematic diagram is drawn. $N_{0}=79.6764+0.2422(Y-1985)-I N T[0.25(Y-1985)]$

Where,

a) Y stands for year;

b) INT (X) stands for the standard function of the maximum integer no larger than X by BASIC language.

Error test:

According to the above equation, EQ is no larger than 30s.

2) True solar time (TT)

Since the Earth orbits the sun elliptically, the rotation period of the Earth to the Sun is not even, being faster sometimes and lower other times. The average rotation speed in a day is called the local average solar time, TM. Within one year, the average daily local solar time differs from the true solar time to some extent. Considering the accuracy of calculation results, the true solar time should be worked out.

The true solar time can be worked out according to the following equation:

$$
\begin{gathered}
T_{M}=C_{r}+L_{i} \\
T T=T_{M}+E_{Q}=C_{r}+L_{c}+E_{Q}
\end{gathered}
$$

Where: 1) TM stands for the average local solar time (local time);

2) CT stands for the local standard time (zone time);

3) LC stands for the rectified value of the longitude (4min/degree). If the local meridian is on the east of the standard meridian, LC is positive; otherwise, it is negative. Below is the equation for calculation:

$$
L_{c}=(D M-120) \times 4 \quad(\mathrm{~min})
$$

Where: DM stands for the longitudinal value of the observation location.

1) Solar declination: $\mathrm{DE}$

Solar declination refers to the included angle between the ligature connecting the sun and the center of the Earth, and the terrestrial equator. The declination angle adopts "year" as its cycle, moving within $+23^{\circ} 26^{\prime}$ and $-23^{\circ} 26^{\prime}$, and becoming a symbol of seasons. It can be calculated according to the following equation: 


$$
\begin{aligned}
D_{E}= & 0.3723+23.2567 \sin Q+0.1149 \sin 2 Q-0.1712 \sin 3 Q \\
& -0.7580 \cos Q+0.3656 \cos 2 Q+0.0201 \cos 3 Q
\end{aligned}
$$

Where, 1) ø stands for the local altitude;

2) $T_{0}$ stands for the solar time angle, which can be worked out through the following equation:

$$
T_{0}=(T T-12) \times 15
$$

3) Solar azimuth, A

Solar azimuth refers to the included angle between the projection of solar rays on the ground and the local longitude, which can be approximately regarded as the included angle between the shadow of the straight line on the ground under the sunlight and the south. As is shown in Fig. 1, it can be calculated according to the following equation:

$$
\sin A=-\cos D_{E} \cdot \sin T_{0} / \sin H_{A}
$$

The potential date differing slightly from the actual value is searched within the year 2015 . MATLAB is employed to work out the corresponding error of $\mathrm{N}$ in every day of 365 :

1) Changing curve of $\mathrm{N}$ along with the error of the shadow length in Attachment 2. (See Fig. 2)

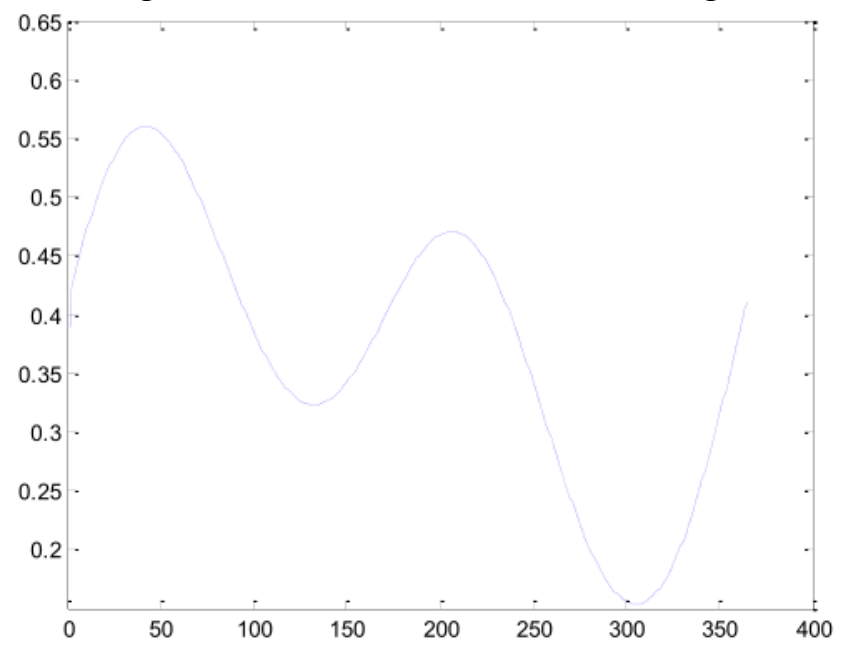

Fig. 2 Changing curve of $\mathrm{N}$ along with the error of the shadow length in Attachment 2

2) Changing curve of $\mathrm{N}$ along with the error of the shadow length in Attachment 3. (See Fig. 3)

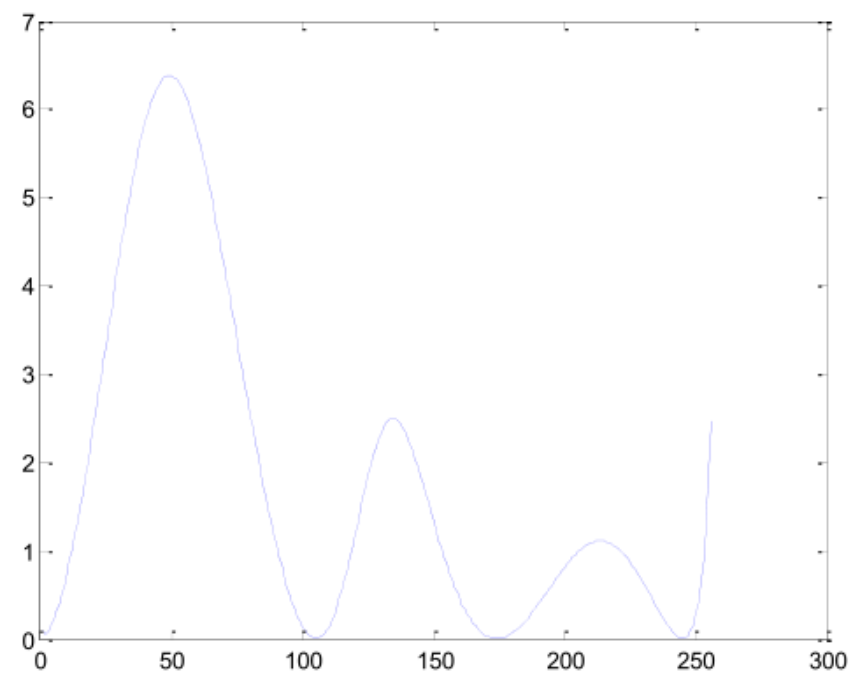

Fig. 3 Changing curve of $\mathrm{N}$ along with the error of the shadow length in Attachment 3

\section{Establishment and solution of the model}

3.1 Confirm the coordinate of the vertex of the shadow in the rectangular coordinate system

1) Confirm the skew coordinate of the solar shadow vertex in the straight bar in the image 
Import videos into MATLAB and adopt 2min as the time interval. Capture images (21 pieces in total) at the designated moment, and import images into AuToCAD. Adopt the center of the foundation as the original point of the coordinate, and the floor tile edge line paved on the horizontal ground in images as the $\mathrm{x}$-coordinate and y-coordinate, based on which the skew coordinate system is built [3]. The skew coordinate of the vertex of the corresponding shadow in every moment in images is obtained, which is $\left(X^{\prime}, Y^{\prime}\right)$, and the straight-bar height in images is $h$.

2) Confirm the rectangular coordinate of the vertex of solar shadow of the straight bar in images

Use CAD to draw the relationship schematic diagram between the skew coordinate system and the rectangular coordinate system. (See Fig. 4)

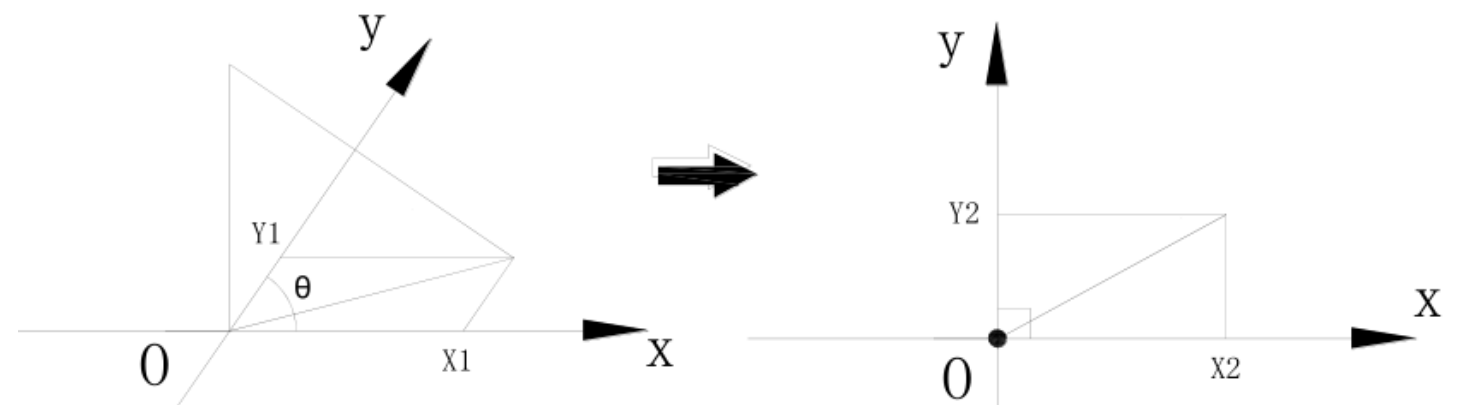

Fig. 4 Relationship schematic diagram between the skew coordinate system and the rectangular coordinate system

The included angle between the skew coordinate system and the rectangular coordinate system measured is shown below:

$\theta=56^{\circ}$

The equation for the conversion of the point on the skew coordinate system to that on the rectangular coordinate system is shown below:

$$
\left\{\begin{array}{l}
X=\left(X^{\prime}-Y^{\prime}\right) \cdot \sin \theta \\
Y=\left(X^{\prime}+Y^{\prime}\right) \cdot \sin \theta
\end{array}\right.
$$

Where,

$\left(\mathrm{X}^{\prime}, Y^{\prime}\right)$ stands for the skew coordinate of the vertex of the straight-bar shadow in images;

$(X, Y)$ stands for the rectangular coordinate of the vertex of the straight-bar shadow in images.

\section{Summary}

In terms of analysis of the photographing location and date, the coordinate of the vertex of the straight-bar shadow actually measured can be put into the model. According to the imaging principle, the ratio of the measured coordinate value to the straight-bar height is equal to the ratio of the practical coordinate value to the straight-bar height. Therefore, videos can be imported into MATLAB and images are captured at the required time interval. CAD is used to measure the skew coordinate of the vertex of the shadow in images. Through the coordinate conversion, it can be converted into the rectangular coordinate in images. Based on the proportional relationship, the coordinate of the vertex of the practical straight-bar shadow can be confirmed. Following that, the model can be employed to confirm the possible photographing location and date for certain video.

\section{References}

[1] Du W S, Li T \& Lu Y B. Improvement and application of solar position equation[J]. Water Resources and Power, 2011 (09).

[2] Du C X, Wang P, Ma C F, Wu Y T \& Shen S Q. A high-precision solar position algorithm[J]. Energy Engineering, 2010 (02).

[3]HowNet: Solar Azimuth[EB/OL].http://epub.cnki.net/kns/brief/default_result.aspx 